\title{
Characterisation of five candidate genes within the ETEC F4ab/ac candidate region in pigs
}

\author{
Mette Jacobsen ${ }^{1}$, Susanna Cirera ${ }^{1}$, David Joller ${ }^{2}$, Gloria Esteso $^{3}$, Steffen S Kracht ${ }^{1}$, Inger Edfors ${ }^{4}$, Christian Bendixen $^{5}$, \\ Alan L Archibald ${ }^{6}$, Peter Vogeli ${ }^{2}$, Stefan Neuenschwander ${ }^{2}$, Hans U Bertschinger ${ }^{2}$, Antonio Rampoldi ${ }^{2}$, \\ Leif Andersson ${ }^{7}$, Merete Fredholm ${ }^{1}$ and Claus B Jørgensen ${ }^{1 *}$
}

\begin{abstract}
Background: Enterotoxigenic Escherichia coli (ETEC) that express the F4ab and F4ac fimbriae is a major contributor to diarrhoea outbreaks in the pig breeding industry, infecting both newborn and weaned piglets. Some pigs are resistant to this infection, and susceptibility is inherited as a simple dominant Mendelian trait. Indentifying the genetics behind this trait will greatly benefit pig welfare as well as the pig breeding industry by providing an opportunity to select against genetically susceptible animals, thereby reducing the number of diarrhoea outbreaks. The trait has recently been mapped by haplotype sharing to a $2.5 \mathrm{Mb}$ region on pig chromosome 13, a region containing 18 annotated genes.

Findings: The coding regions of five candidate genes for susceptibility to ETEC F4ab/ac infection (TFRC, ACK1, MUC20, MUC4 and KIAA0226), all located in the $2.5 \mathrm{Mb}$ region, were investigated for the presence of possible causative mutations. A total of 34 polymorphisms were identified in either coding regions or their flanking introns. The genotyping data for two of those were found to perfectly match the genotypes at the ETEC F4ab/ac locus, a G to $C$ polymorphism in intron 11 of TFRC and a C to T silent polymorphism in exon 22 of KIAA0226. Transcriptional profiles of the five genes were investigated in a porcine tissue panel including various intestinal tissues. All five genes were expressed in intestinal tissues at different levels but none of the genes were found differentially expressed between ETEC F4ab/ac resistant and ETEC F4ab/ac susceptible animals in any of the tested tissues.

Conclusions: None of the identified polymorphisms are obvious causative mutations for ETEC F4ab/ac susceptibility, as they have no impact on the level of the overall mRNA expression nor predicted to influence the composition of the amino acids composition. However, we cannot exclude that the five tested genes are bona fide candidate genes for susceptibility to ETEC F4ab/ac infection since the identified polymorphism might affect the translational apparatus, alternative splice forms may exist and post translational mechanisms might contribute to disease susceptibility.
\end{abstract}

\section{Background}

Diarrhoea in neonatal and newly weaned pigs is a serious welfare problem and a financial burden in pig production. Some piglets are resistant to certain types of bacterial infection and identifying the genetic cause for susceptibility is a prerequisite for an effective selection program. One of the bacteria causing diarrhoea is enterotoxigenic Escherichia coli (ETEC) that expresses the F4 fimbriae with the variants F4ab, F4ac and F4ad. These

\footnotetext{
* Correspondence: chj@life.ku.dk

'Department of Basic Animal and Veterinary Sciences, University of

Copenhagen, 1870 Frederiksberg, Denmark

Full list of author information is available at the end of the article
}

fimbriae adhere particularly to specific receptors on the brush borders of the enterocytes of the small intestine [1]. The enterocytes of pigs susceptible to ETEC F4ab and F4ac diarrhoea express a receptor that interacts with F4ab and F4ac adhesins, and the expression of this receptor is inherited as an autosomal dominant Mendelian trait [2]. We therefore expect that the candidate region for susceptibility to ETEC F4ab/ac infection contains a gene encoding the receptor itself, an endogenous ligand or a product which modifies the structure and function of the receptor.

The locus responsible for susceptibility has been mapped to pig chromosome 13 in the q41 region by two

\section{Biomed Central}


independent linkage studies $[3,4]$ and the candidate region has subsequently been narrowed down to $5.7 \mathrm{cM}$ [5]. Recently, the locus has been further refined by haplotype sharing to a region of $3.1 \mathrm{Mb}$ [6]. This region corresponds to approximately $2.5 \mathrm{Mb}$ in the published pig map in Ensembl (Sscrofa9), from position 100.680.954 to 103.192.935, and contains 18 annotated porcine genes.

Protein-protein interaction studies have revealed that the F4ab/ac receptor, which interacts with the F4ab/ac fimbriae, is most likely to be either a mucin-like sialoglycoprotein or a transferrin-like protein [7-9]. Previously, intron 7 of the mucin 4 gene (MUC4) was shown to contain a non-causative polymorphism in complete LD with susceptibility to ETEC infection [10]. Five candidate genes in the $2.5 \mathrm{Mb}$ region including and in the vicinity of MUC4 were therefore screened for mutations in the coding sequences and in parts of the intervening introns in order to identify possible genetic alterations in ETEC F4ab/ac susceptible animals. In addition to MUC4 these genes were the transferrin receptor (TFRC), the tyrosine kinase, non-receptor, 2 (ACK1), the mucin 20 (MUC20) and the KIAA0226 genes. Both MUC4 and MUC2O are striking candidate genes for susceptibility, as both are membrane bound, highly glycosylated proteins, and contribute to the immune defence system by being major components of the glycocalyx layer of epithelial cells. In addition, they are both highly abundant in the gastrointestinal tract $[11,12]$. TFRC is essential for the transport of iron from the transferrin protein into the cell, and E. coli bacteria are dependent on iron availability for survival and propagation. The cDNA sequence for TFRC has previously been explored for mutations associated with ETEC F4ab/ac susceptibility [13,14], but none of these studies explored the exon-intron junctions for mutations that could interfere with proper splicing of the transcript. ACK1 has been shown to impact the expression level of TFRC [15], thereby making $A C K 1$ a potential candidate gene. Little is known about the protein encoded by KIAA0226, called Rundataxin due to the presence of a RUN element in the primary sequence and its association with the human disease Ataxia [16].

Since animals susceptible to ETEC infection express the F4ab/ac receptor irrespective of the presence of bacteria and since susceptibility might be reflected at the level of gene transcription, we investigated the transcriptional profile by quantitative real time PCR (qPCR) of the five candidate genes in 11 different tissues and intestinal cells from both ETEC F4ab/ac susceptible and resistant animals. The research hypothesis behind selection of the five invested candidate genes for ETEC F4ab/ac susceptibility was two-fold: 1) Their location in a region closely linked to susceptibility and 2) the ontology of the encoded proteins.

\section{Methods}

\section{Animals}

Investigation of the genetic variations in the five candidate genes, TFRC, ACK1, MUC4, MUC20 and KIAA0226, was performed on genomic DNA from two resistant Wild Boars and two homozygous susceptible Large White sows. All four animals were phenotyped for both F4ab and F4ac adhesion [1] and have previously been described [6].

Additional genotyping of the six identified polymorphisms were performed on genomic DNA from a total of 42 pigs; six susceptible Large White sows, two susceptible and four resistant Yorkshire pigs, one susceptible Landrace and one resistant Landrace pig, 26 susceptible and two resistant crossbreeds between Duroc, Landrace and Yorkshire. They were all phenotyped for both F4ab and F4ac adhesion as previously described $[1,4]$

For the expression profiles, four 3-months old siblings were slaughtered ( 2 female and 2 male crossbreeds between Landrace, Yorkshire and Duroc), and 11 tissues (lung, liver, kidney, lymph nodes, muscle, pancreas, cerebral cortex, colon, jejunum, duodenum and ileum) were collected, immediately frozen in liquid nitrogen and stored at $-80^{\circ} \mathrm{C}$ until RNA purification. No information on F4ab/ ac status was available from these animals.

Furthermore, expression profiles of the candidate genes in enterocytic cells from jejunum were investigated in 5 resistant and 5 susceptible Yorkshire animals that were phenotyped for both F4ab and F4ac adhesion as previously described [1].

All samples were collected from animals kept under conditions required for farm animals in Denmark. No approvals from ethics committees were required for this study.

\section{Extraction of DNA and RNA}

DNA was extracted from whole blood using the Epicenter kit (Promega) according to the manufacturer's protocol. The amount and quality of DNA was measured using Nanodrop 1000 (Thermo Fisher Scientific). Total RNA was extracted from tissue samples (two biological replicates per tissue and animal) using TriReagent ${ }^{\circledR}$ (Molecular Research Centre), according to the manufacturer's recommendations. After purification the RNA was DNase treated using spin-column purification (Qiagen). Total RNA was quantified by optical density and the quality was evaluated by ribosomal RNA $28 \mathrm{~S} / 18 \mathrm{~S}$ band inspection by gel electrophoresis and by RNA integrity number (RIN, 2100 Bioanalyzer, Agilent technologies). A RIN number of five was chosen as minimum threshold for acceptance.

\section{Primer design, PCR and sequencing}

Using mRNA sequences from either human and/or pig of the five candidate genes TFRC [GenBank: NM_214001], 
ACK1 [GenBank: NM_001010938 and GenBank: NM_005781], MUC4 [GenBank: NM_018406], MUC20 [GenBank: NM_001113440] and KIAA0226 [GenBank: NM_001145642 and NM_014687] to blast against the porcine sequenced BAC-clones [GenBank: CU695181, CU914410, CU468995, CU694544 and FP102930], it was possible to identify all the genomic coding sequences in the pig, except for TFRC exon 1, ACK1 exon 1, MUC4 exon 2 and KIAA0226 exon 1. Primers were designed in intron sequences flanking the exons using the PRIMER3 software http://frodo.wi.mit.edu. For a complete list of primer sequences, amplicon sizes, and coverage, see additional file 1 . The primer pairs were all tested for amplification efficiency over a range of annealing temperatures using TEMPase Hot Start DNA polymerase and amplicons were amplified according to the manufacturer's recommendations (Amplicon). $5 \mu \mathrm{l}$ of the PCR products were visualised on a $1 \%$ agarose gel and the remaining PCR products were purified using Montage $\mathrm{PCR}_{96}$ Cleanup kit (Millipore), and sequenced using BigDye Terminator Cycle Sequencing Kit (Applied Biosystems) according to the manufacturer's recommendations. Sequenced products were purified with Montage SEQ 96 Cleanup kit (Millipore), and resolved on an ABI3130 $\times 1$ Genetic Analyzer (Applied Biosystems). Traces were assembled and visualised using the LaserGene software V.7.2 (DNASTAR).

\section{5' and $3^{\prime}$ RACE}

To obtain reliable sequence data of the 5' and 3'ends of porcine MUC4 and the 5'end of porcine $A C K 1$, the SMARTTM RACE cDNA amplification kit from Clontech was used. Three primers with a melting temperature of approximately $70^{\circ} \mathrm{C}$ were designed in exon 3 of $A C K 1$ and in exons 1 and 24 of MUC4 [Additional file 1], and used in the second strand cDNA synthesis according to the manufacturer's protocol (Clontech). It was possible to amplify three fragments of approximately $400 \mathrm{bp}$ (ACK1 5'UTR), $800 \mathrm{bp}$ (MUC4 3'UTR) and $250 \mathrm{bp}$ (MUC4 5'UTR), respectively. Fragments were purified using a gel purification kit (Qiagen) according to the manufacturer's recommendations. The purified amplicons were then ligated into pGEM $^{\circledR}$-T Easy Vectors (Promega) and cloned into JM109 High Efficiency Competent Cells (Promega). M13 forward and M13 reverse primers were used in a sequencing reaction similar to that described above.

\section{Analysis of identified polymorphisms}

The individual polymorphisms were tested for association with the ETEC F4ab/ac adhesion phenotypes using a chisquared test, and a standardised linkage disequilibrium value (D') were calculated using Haploview 4.1 [17]. Prediction of splice defects was performed in Human Splice finder - Version 2.4.1 http://www.umd.be/HSF/ [18], where the genomic regions of the TFRC and KIAA0226 genes containing the two polymorphisms in LD with susceptibility to infection (numbers 3 and 34) were exposed to mutation analysis using default settings.

\section{Real time quantitative RT-PCR (qPCR)}

One $\mu \mathrm{g}$ of each total RNA sample was reverse transcribed at $42^{\circ} \mathrm{C}$ using a mixture of Oligo $(\mathrm{dT})$ and random hexamers (1:3) and Improm-II ${ }^{\mathrm{TM}}$ reverse transcriptase (Promega) according to the manual. All primers were designed using PRIMER3 software. Two $\mu \mathrm{l}$ of each cDNA sample (diluted 1:8) was then added to the PCR mixture, consisting of $5 \mu \mathrm{l}$ QuantiFast ${ }^{\mathrm{TM}}$ SYBR Green master mix (Qiagen) and $0.5 \mathrm{mM}$ of each primer (See Table 1 for primer list). All reactions were performed in an Mx3000PTM machine (Stratagene). Thermal cycling conditions were $95^{\circ} \mathrm{C}$ for $5 \mathrm{~min}$, followed by 40 cycles at $95^{\circ} \mathrm{C}$ for $15 \mathrm{~s}$ and $60^{\circ} \mathrm{C}$ for $30 \mathrm{~s}$. A dissociation curve was made $\left(95^{\circ} \mathrm{C}\right.$ for $1 \mathrm{~min}, 55^{\circ} \mathrm{C}$ for $30 \mathrm{~s}$, and $95^{\circ} \mathrm{C}$ for $30 \mathrm{~s}$ ) to confirm the specificity of the primers.

Expression levels of the five candidate genes were normalised across tissues using three stable reference genes TBP, HMBS and RPL4 [19]. The GeNorm software [20] was used to calculate a normalisation factor (NF). Samples were normalised by the NF and fold changes were calculated. Subsequently, the normalised fold changes were tested for normal distribution within each tissue using Instat 3.0 (GraphPad). One factor Analysis of Variance (ANOVA) statistical test was performed to test for significant differences.

\section{Results \\ Porcine orthologues of TFRC, ACK1, MUC4, MUC20 and KIAA0226}

The coding parts of each of the five genes were sequenced and data submitted to GenBank with the following accession numbers; TFRC [GenBank: HM070995], ACK1 [GenBank: HM070993], MUC4 [GenBank: GU983681], MUC20 [GenBank: HM070996] and KIAA0226 [GenBank: HM849042]. All exons contain consensus donor/acceptor splice sites, and are almost identical in size to their human orthologues, except for the porcine MUC20 gene whose exon 2 is orthologues to human exons 2 and 3 combined. However, exon 2 of porcine $A C K 1$ differs significantly from human $A C K 1$ exon 2 by encoding only 21 amino acids as opposed to 57 amino acids in human.

All exons, except for TFRC exon $1, A C K 1$ exon 1 , KIAA0226 exon 1, and exon 2 of MUC4, were investigated for the presence of mutations. The coding part of human TFRC begins in exon 2, and due to the sequence diversity in the 5'UTR between human and pig, it was not possible to accurately predict or amplify TFRC exon 
Table 1 Identified polymorphisms in porcine TFRC, ACK1, MUC4, MUC2O and KIAA0226 genes

\begin{tabular}{|c|c|c|c|}
\hline Gene & No. & Base & Consequence \\
\hline \multirow[t]{3}{*}{ TFRC (100.8 Mb) } & 1 & $\mathrm{G} \rightarrow \mathrm{A}(\mathrm{E} 4)$ & $\mathrm{Ala} \rightarrow \mathrm{Thr}$ \\
\hline & 2 & $T \rightarrow C(E 10)$ & Silent \\
\hline & 3 & $\mathrm{G} \rightarrow \mathrm{T}(\mathrm{I11})$ & 10 bp before branch point \\
\hline \multirow[t]{17}{*}{ ACK1 (100.9 Mb) } & 4 & $C \rightarrow G(E 1)$ & Pro $\rightarrow$ Ala \\
\hline & 5 & $\mathrm{~T} \rightarrow \mathrm{C}(\mathrm{E} 2)$ & Silent \\
\hline & 6 & $\mathrm{G} \rightarrow \mathrm{T}(\mathrm{E} 2)$ & Silent \\
\hline & 7 & $C \rightarrow T(E 3)$ & Silent \\
\hline & 8 & $A \rightarrow C(E 7)$ & Silent \\
\hline & 9 & $C \rightarrow T(E 7)$ & Silent \\
\hline & 10 & $\mathrm{~T} \rightarrow \mathrm{C}(\mathrm{E} 9)$ & Silent \\
\hline & 11 & $C \rightarrow T(E 9)$ & Silent \\
\hline & 12 & $G \rightarrow A(E 10)$ & Silent \\
\hline & 13 & $C \rightarrow A(E 12)$ & Pro $\rightarrow$ His \\
\hline & 14 & $A \rightarrow G(E 12)$ & Silent \\
\hline & 15 & $\mathrm{G} \rightarrow \mathrm{A}(\mathrm{E} 12)$ & Silent \\
\hline & 16 & $\mathrm{G} \rightarrow \mathrm{A}(\mathrm{E} 12)$ & $\mathrm{Val} \rightarrow$ Met \\
\hline & 17 & $A \rightarrow G(E 12)$ & Silent \\
\hline & 18 & $\mathrm{G} \rightarrow \mathrm{A}(\mathrm{E} 12)$ & $\mathrm{Arg} \rightarrow \mathrm{His}$ \\
\hline & 19 & $\mathrm{G} \rightarrow \mathrm{C}(\mathrm{E} 12)$ & $\mathrm{Ser} \rightarrow \mathrm{Thr}$ \\
\hline & 20 & $C \rightarrow G(114)$ & 5 bases before exon 15 \\
\hline \multirow[t]{9}{*}{ MUC4 (101.0 Mb) } & 21 & $C \rightarrow A(E 12)$ & Silent \\
\hline & 22 & $\mathrm{C} \rightarrow \top(\mid 13)$ & 4 bases before exon 14 \\
\hline & 23 & $C \rightarrow \top(113)$ & 16 bases before exon 14 \\
\hline & 24 & $C \rightarrow \top(113)$ & 28 bases before exon 14 \\
\hline & 25 & $C \rightarrow \top(E 19)$ & Silent \\
\hline & 26 & $G \rightarrow A(E 24)$ & Gly $\rightarrow$ Ser \\
\hline & 27 & $\mathrm{~T} \rightarrow \mathrm{C}(\mathrm{E} 24)$ & Silent \\
\hline & 28 & $\mathrm{G} \rightarrow \mathrm{A}(3 \cup T R)$ & 187 bases after TGA \\
\hline & 29 & $\mathrm{G} \rightarrow \mathrm{A}(3 \cup T R)$ & 259 bases after TGA \\
\hline \multirow[t]{4}{*}{ MUC20 (101.1 Mb) } & 30 & $\mathrm{~A} \rightarrow \mathrm{C}(3 \mathrm{UTTR})$ & 201 bases after TGA \\
\hline & 31 & $\mathrm{C} \rightarrow \mathrm{T}(\mathrm{I})$ & 58 bases after exon 2 \\
\hline & 32 & $C \rightarrow T(E 2)$ & Silent \\
\hline & 33 & $\mathrm{~A} \rightarrow \mathrm{T}(\mathrm{E} 1)$ & Silent \\
\hline KIAA0226 (101.1 Mb) & 34 & $\mathrm{C} \rightarrow \mathrm{T}(\mathrm{E} 20)$ & $\underline{\text { Silent }}$ \\
\hline
\end{tabular}

A total of 34 polymorphisms were identified in the four tested animals ( 2 resistant Wild Boars and 2 homozygous susceptible Large White sows). Gene names are shown in the first column (gene positions at chromosome 13 in Sscrofa 9 are in brackets), polymorphisms in the second column and E and I in brackets indicate exonic (E) or intronic (I) location of the polymorphisms. The third column shows the nature of the polymorphisms. The six polymorphisms matching the ETEC F4ab/ac genotype perfectly in the four animals are in bold, and the two polymorphisms underlined are also closely associated with the ETEC F4ab/ac status in the additional 42 animals.

1 in the pig genome. The same was true for $A C K 1$ exon 1 , and this exon was therefore not identified in the porcine genome either. Human KIAA0226 contains two distinct splice variants [GenBank: NM_014687 and NM_001145642], of which splice variant 1 represents the longest transcript where the first exon is part of the 5 'UTR. Due to the sequence diversity in this region, it was not possible to identify exon 1 of KIAA0226 by similarity search.
Exon 2 of MUC4 contains a 456 base pair sequence, which has been shown to be repeated between 13-21 times in eight pigs from four different breeds (Landrace, Yorkshire, Hampshire and Duroc) according to a Southern blot made by our group (data not shown). This region of the MUC4 gene is therefore too large (averaging $8 \mathrm{~kb}$ ) and complex and thus not possible to analyse using capillary sequencing on PCR product. As MUC4 is an obvious prime candidate gene for susceptibility to infection by ETEC F4ab/ac, we identified the whole 5' and 3' untranslated regions using Rapid Amplification of cDNA Ends (RACE).

A total of 34 polymorphisms were identified in the 5 tested genes in the four animals, two F4ab/ac resistant Wild Boars and two F4ab/ac homozygous susceptible Large White sows. 25 polymorphisms were present in the coding regions and nine in the intron sequences (Table 1). All polymorphisms have been annotated in the submitted sequences in GenBank.

Seven of the 25 coding polymorphisms are predicted to result in amino acids changes (Table 1 ), but none of them can be exclusively associated with the F4ab/ac phenotype in the four tested animals. However, the genotypes at six polymorphic sites matched perfectly the ETEC F4ab/ac phenotypes in these animals (Table 1, bold). These six SNPs were subsequently genotyped in 42 additional and phenotyped animals, and very tight association with the susceptible F4ab/ac phenotype was observed for two of the SNPs; polymorphisms number 3 and 34 (Table 1, underlined and bold).

\section{Transcription profiling}

To investigate the expression profiles of the five candidate genes, real-time qPCR was performed using total RNA from 11 different tissues. These tissues originate from four piglets of crosses between the Landrace, the Yorkshire and the Duroc breeds. No information of their ETEC F4ab/ac susceptibility status is known. The expression profiles of the five genes were normalised to three internal controls; TBP, HMBS and RPL4 [19]. All five genes were expressed in all tissues examined, but at markedly different levels (Table 2). TFRC has the highest expression in muscle, and is expressed in all four intestinal tissues at a relatively high level. $A C K 1$ has a significantly higher expression level in cerebral cortex than in other tissues $(\mathrm{P}<0.001)$, but is still expressed at moderate levels in the four intestinal tissues. MUC4 is predominantly expressed in colon $(\mathrm{P}<0.0001)$, but present at a low level in all other tissues. MUC20 has the highest expression in duodenum $(\mathrm{P}<0.05)$ and kidney $(\mathrm{P}<0.05)$, but is also expressed in the three other intestinal tissues at a relatively high level. KIAA0226 shows an expression profile quite similar to the expression profile of $A C K 1$, with the highest expression in cerebral cortex, and moderate expression in all other tissues. 
Table 2 Expression profile of the porcine TFRC, ACK1, MUC4, MUC20 and KIAA0226 gene

\begin{tabular}{llllll}
\hline & TFRC & ACK1 & MUC4 & MUC20 & KIAA0226 \\
\hline Cerebral cortex & 68.1 & 100.0 & 0.2 & 3.1 & 100.0 \\
Colon & $\mathbf{5 1 . 9}$ & $\mathbf{1 7 . 1}$ & $\mathbf{1 0 0 . 0}$ & $\mathbf{2 2 . 1}$ & $\mathbf{2 6 . 5}$ \\
Duodenum & $\mathbf{6 7 . 2}$ & $\mathbf{6 . 9}$ & $\mathbf{8 . 2}$ & $\mathbf{1 0 0 . 0}$ & $\mathbf{2 5 . 2}$ \\
Ileum & $\mathbf{2 9 . 3}$ & $\mathbf{1 0 . 8}$ & $\mathbf{4 . 5}$ & $\mathbf{3 8 . 3}$ & $\mathbf{3 9 . 9}$ \\
Jejunum & $\mathbf{3 6 . 6}$ & $\mathbf{7 . 5}$ & $\mathbf{1 . 0}$ & $\mathbf{1 6 . 6}$ & $\mathbf{3 0 . 8}$ \\
Kidney & 56.1 & 11.3 & 1.1 & 71.6 & 32.7 \\
Liver & $\mathbf{6 2 . 1}$ & 50.4 & 0.3 & 4.4 & 21.3 \\
Lung & 21.5 & 38.3 & 1.6 & 31.0 & 76.7 \\
Lymph notes & 25.5 & 33.9 & 0.2 & 2.1 & 45.4 \\
Muscle & 100.0 & 19.5 & 1.2 & 1.5 & 25.6 \\
Pancreas & 32.6 & 21.5 & 3.7 & 25.7 & 12.9 \\
\hline
\end{tabular}

The expression profiles of the five genes in 11 porcine tissues from four animals (crossbreeds between Landrace, Yorkshire and Duroc). The expression levels are normalised to 3 internal reference genes and scaled to 100 for the tissue with highest expression. The levels can be compared between tissues but not between genes. Expression levels in the four intestinal tissues are in bold.

The expression levels of the five genes in enterocytes from jejunum from a total of 10 Yorkshire animals phenotyped as either susceptible or resistant to F4ab/ac infection (five of each) were measured by qPCR. ANOVA test of the qPCR data did not reveal any significant differential expression of the five candidate genes between susceptible and resistant animals (Figure 1).

\section{Discussion}

Infection by enterotoxigenic Escherichia coli (ETEC) $\mathrm{F} 4 \mathrm{ab} / \mathrm{ac}$ is a major cause of death in piglets and identification of the causative gene(s) will have an impact on breeding programs. By selecting for resistant animals the cost for the pig production could be reduced and

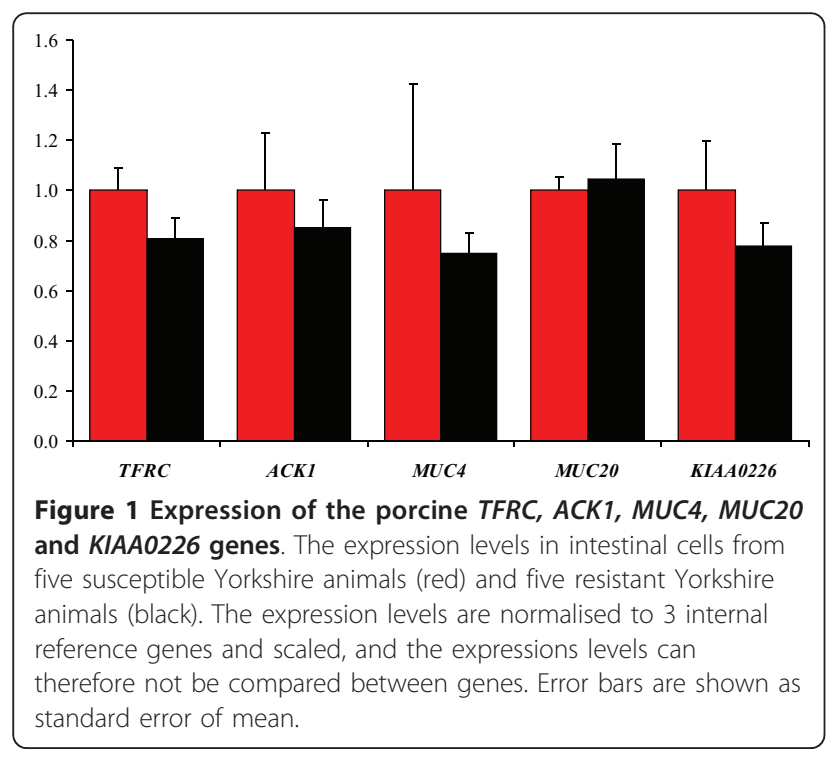

livestock welfare would be increased. Susceptibility to this infection is inherited as a Mendelian dominant trait, and we characterised the porcine MUC4 gene and the four neighbouring genes for exonic and intronic mutations. The localisation of these investigated five genes on pig chromosome 13 is depicted in figure 2. A total of six polymorphisms matched perfectly the ETEC F4ab/ac genotype in four animals of the Large White breed and Wild Boars, and these six polymorphisms were genotyped in 42 additional animals of different breeds. Two of these, polymorphisms 3 and 34, were shown to be significantly associated with the F4ab/ac phenotype with chisquares of 26.2 and 31.7 , respectively. These two polymorphisms are located in either an exon or an intronsequence in close proximity to an exon. One polymorphism in intron 11 of TFRC (No. 3) is located 61 bases upstream of exon 12, and one CTG-TTG synonymous substitution is located in the last exon of KIAA0226 (No. 34). These polymorphisms are not obvious causative mutations for ETEC F4ab/ac susceptibility, but they are both predicted to either introduce or disrupt a splice regulatory sequence using Human Splicing Finder V2.4 [18]. The cDNA sequence for TFRC has been investigated previously using both animals resistant and susceptible to ETEC infection $[13,14]$ but no evidence of splice variants were reported. The intron 11 polymorphism is thus not likely to influence the regulation of splicing of the primary messenger RNA. As for the KIAA0226 gene, we have compared sequences from genomic and cDNA reads and have not found evidence of either introduction/or skipping of exons in relation to the ETEC F4ab/ ac status. However it can not be excluded that one of these two polymorphisms are located in a regulatory region, necessary for proper translation, thereby affecting the level of protein synthesis.

An LD test has revealed a D' value of 0.94 between polymorphisms 3 and 34, indicating a tight association, where the polymorphism in KIAA0226 shows the highest degree of association with the ETEC F4ab/ac phenotype (q-square value of 31.7 ). Several studies using various pig breeds have indentified markers in the candidate region that are tightly associated, suggesting that the whole region between and including the TFRC and $M U C 13$ genes is in tight linkage disequilibrium with the ETEC F4ab/ac locus $[5,10,14,21,22]$. However, the polymorphism in intron 7 of MUC4, which is in complete LD with the ETEC F4ab/ac locus in both a Swiss and Swedish porcine population [5], is only associated and not in complete LD with the F4ab/ac locus in a Belgian breed [23]. A similar conclusion was reached in a Chinese study [24]. These discrepancies are due to a high degree of LD in the candidate region, the use of different breeds and populations and uncertainties in relation to the phenotyping methods. 


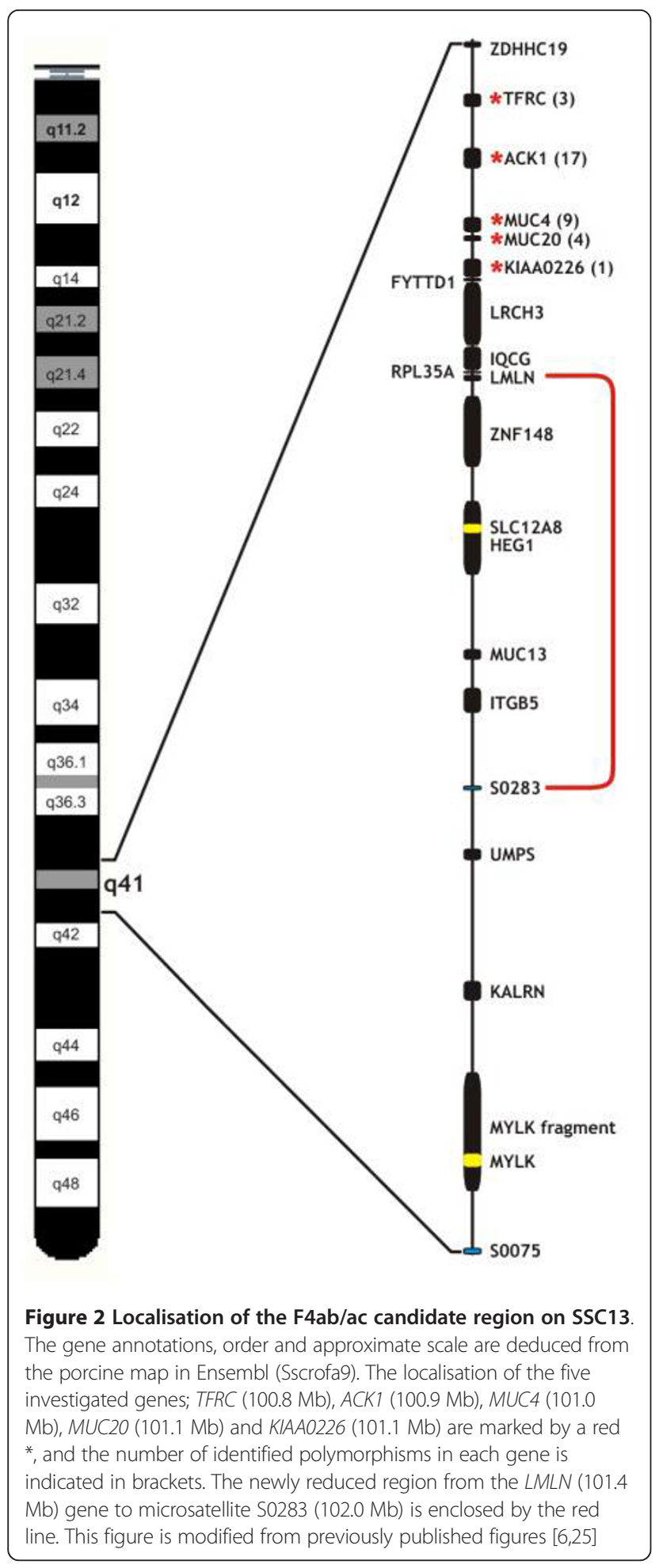

Recently, a recombination event was observed in a Swiss boar, which reduces the candidate region considerably. This region now maps from $L M L N$ to $\mathrm{S} 0283$ (Figure 2), distal to KIAA0226, thereby excluding the five candidate genes analysed here from the candidate region [25]. However these data are all based on a small population and a more comprehensive study of this recombination event is needed for clarification.

Since animals susceptible to ETEC infection express the F4ab/ac receptor irrespective of the presence of the bacteria and since susceptibility might be reflected at the level of gene transcription, we investigated the transcriptional profiles of the five candidate genes. We constructed an expression panel consisting of various intestinal tissues, muscle and other organs in order to compare the expression patterns across tissues. All five genes were expressed in the intestinal tissues, although the expression of MUC4 was very low in all tissues tested when compared to the expression in colon. No significant differences were found between the expression levels in F4ab/ac phenotyped resistant and susceptible animals. This confirms an earlier report on expression of the $M U C 20$ gene in pigs infected with ETEC F4ac [26].

A comprehensive cDNA analysis has been performed on ACK1, MUC4 and KIAA0226. Two transcript variants are known for human $A C K 1$. The most highly expressed transcript variant 1 [GenBank: NM_005781] encompass all exons except for exons 2 and 13. Transcript variant 2 [GenBank: NM_001010938] contains an alternative 5 'UTR with a start codon in exon 2 , and it does not contain exon 15. We identified exon 2 of $A C K 1$ using RACE, which suggests the presence of the rarer transcript variant 2 in the pig. However, we failed to identify exon 13 and no published ESTs seem to exist for this exon. We can thus not conclude if pigs contain two transcripts of $A C K 1$ similar to humans or if porcine $A C K 1$ is different from any of the human transcripts.

Several variants of human MUC4 have been reported from mainly tumour tissues and transcript variants 1,4 and 5 are commonly observed. Transcript variant 1 [GenBank: NM_018406] encompass the full-length transcript, transcript variant 4 [GenBank: NM_004532] lacks exon 2, which contains a large variable tandem repeat region. Transcript variant 5 [GenBank: NM_138297] lacks both exons 2 and 3. Using primers located in porcine MUC4 exon 1 and exon 5-6, it was possible to amplify a specific product from cDNA containing exons 1, 3-5 (data not shown). This suggests the presence of a porcine transcript variant, which is dissimilar to human transcript variants 1 and 5 , and possibly similar to human transcript variant 4. By extending the elongation time during the PCR cycle ( $1 \mathrm{~min}$. to $6 \mathrm{~min}$.), it was also possible to obtain several products differing approximately $450 \mathrm{nt}$. in size, visualised as ladder bands on an agarose gel. Sequencing these products revealed the presence of one to four repeats of $456 \mathrm{nt}$., which suggests that pigs also express an orthologue of human MUC4 transcript variant 1. 
Two transcript variants for the human KIAA0226 gene have been reported. The longest transcript variant 1 [GenBank: 001145642] lacks exon 2, and transcript variant 2 contains an alternative first exon (exon 2) and lacks exon 9. The whole coding region of porcine KIAA0226 was amplified from cDNA and there was no evidence of the presence of exon 9, suggesting that the porcine KIAA0226 transcript is similar to human transcript variant 2 .

These studies of the coding regions of the three genes were all performed on cDNA generated from total RNA purified from jejunum tissue. The absent transcript variants could therefore still be present in others tissues, and to exclusively confirm the number of transcript variants of these genes, a northern blot is needed, and preferably from several different porcine tissues. In this study the overall expression levels of the five genes have been measured by QPCR using primers located in exons, which are present in all of the known transcript variants. This approach, however, introduces the bias that differences in gene expression between two transcript variants will not be detected.

\section{Conclusion}

Gene characterisation of the porcine orthologues of human genes; TFRC, ACK1, MUC4, MUC2O and KIAA0226 contributes to both the assembly and annotation of the porcine genome, and is beneficial to future studies of these genes in pig. Almost all coding parts of the candidate genes have been investigated for mutations. Although none of the identified polymorphisms are obvious causative candidate mutations, we cannot exclude that these genes are bona fide candidates for susceptibility to ETEC F4ab/ac infection. We have not detected any differences in expression levels of the tested candidate genes between resistant and susceptible animals, but we cannot exclude that alternative splice forms exist or that post translational mechanisms contribute to disease susceptibility.

\section{Additional material}

Additional file 1: Complete list of primer sequences, amplicon sizes and amplicon coverage.

\footnotetext{
Acknowledgements

Thanks to Minna Jacobsen, Christel A. Halberg and Tina N. Mahler for their excellent technical support and to Dr. Per Horn for critically revising the manuscript. This work was supported by the EU project SABRE.

\section{Author details}

${ }^{1}$ Department of Basic Animal and Veterinary Sciences, University of Copenhagen, 1870 Frederiksberg, Denmark. ${ }^{2}$ Institute of Agricultural Sciences, ETH Zurich, 8092 Zurich, Switzerland. ${ }^{3}$ Genomica y Mejora Animal, Departamento de Genética, Universidad de Córdoba, Campus de Rabanales,
}

14071, Córdoba, Spain. ${ }^{4}$ School of Natural Sciences, Linnaeus University, 391 82 Kalmar, Sweden. ${ }^{5}$ Department of Genetics and Biotechnology, Aarhus University, 8830 Tjele, Denmark. 'Division of Genetics and Genomics, The Roslin Institute and R(D)SVS, University of Edinburgh, Roslin, Midlothian EH25 9PS, UK. ${ }^{7}$ Department of Medical Biochemistry and Microbiology, Uppsala University, 75123 Uppsala, Sweden.

\section{Authors' contributions}

MJJ has been involved in all aspects of planning and performing this study, including drafting this manuscript. SC performed the $\mathrm{QPCR}$, and participated in the project coordination together with $C B, L F, M F$ and CBJ. DJ, IE, PV, SN, HUB and $A R$ are responsible for phenotyping of the animals and GE and SSK were involved in the gene characterisation. All authors read and approved the final manuscript.

\section{Competing interests}

The authors declare that they have no competing interests.

Received: 1 February 2011 Accepted: 30 June 2011

Published: 30 June 2011

\section{References}

1. Edfors-Lilja I, Petersson H, Gahne B: Performance of pigs with or without the intestinal receptor for Escherichia coli K88. Anim Prod 1986, 42:381-387.

2. Gibbons RA, Sellwood R, Burrows M, Hunter PA: Inheritance of resistance to neonatal $E$. coli diarrhoea in the pig: Examination of the genetic system. Theor Appl Genet 1977, 51:65-70.

3. Jørgensen CB, Cirera S, Anderson SI, Archibald AL, Raudsepp T, Chowdhary B, Edfors-Lilja I, Andersson L, Fredholm M: Linkage and comparative mapping of the locus controlling susceptibility towards E. COLI F4ab/ac diarrhoea in pigs. Cytogenet Genome Res 2003, 102:157-162.

4. Python $P$, Jorg $H$, Neuenschwander S, Hagger C, Stricker C, Burgi E, Bertschinger HU, Stranzinger G, Vogeli P: Fine-mapping of the intestinal receptor locus for enterotoxigenic Escherichia coli F4ac on porcine chromosome 13. Anim Genet 2002, 33:441-447.

5. Joller D, Jorgensen CB, Bertschinger HU, Python P, Edfors I, Cirera S, Archibald AL, Burgi E, Karlskov-Mortensen P, Andersson L, Fredholm M, Vogeli P: Refined localization of the Escherichia coli F4ab/F4ac receptor locus on pig chromosome 13. Anim Genet 2009, 40:749-752.

6. Jacobsen M, Kracht SS, Esteso G, Cirera S, Edfors I, Archibald AL, Bendixen C, Andersson L, Fredholm M, Jørgensen CB: Refined candidate region specified by haplotype sharing for Escherichia coli F4ab/F4ac susceptibility alleles in pigs. Anim Genet 2010, 41:21-25.

7. Francis DH, Grange PA, Zeman DH, Baker DR, Sun R, Erickson AK: Expression of mucin-type glycoprotein K88 receptors strongly correlates with piglet susceptibility to K88(+) enterotoxigenic Escherichia coli, but adhesion of this bacterium to brush borders does not. Infect Immun 1998, 66:4050-4055.

8. Grange PA, Erickson AK, Anderson TJ, Francis DH: Characterization of the carbohydrate moiety of intestinal mucin-type sialoglycoprotein receptors for the K88ac fimbrial adhesin of Escherichia coli. Infect Immun 1998, 66:1613-1621.

9. Grange PA, Mouricout MA, Levery SB, Francis DH, Erickson AK: Evaluation of receptor binding specificity of Escherichia coli K88 (F4) fimbrial adhesin variants using porcine serum transferrin and glycosphingolipids as model receptors. Infect Immun 2002, 70:2336-2343.

10. Jørgensen CB, Cirera S, Archibald AL, Andersson L, Fredholm M, EdforsLilja I: Porcine polymorphisms and methods for detecting them. International application published under the patent cooperation treaty (PCT) , PCT/DK2003/000807 and WO2004/048606-A2.

11. Linden SK, Florin TH, McGuckin MA: Mucin dynamics in intestinal bacterial infection. PLoS One 2008, 3:e3952.

12. Dharmani $P$, Srivastava $V$, Kissoon-Singh $V$, Chadee K: Role of intestinal mucins in innate host defense mechanisms against pathogens. J Innate Immun 2009, 1:123-135.

13. Python $P$, Jorg $H$, Neuenschwander S, Asai-Coakwell M, Hagger C, Burgi E, Bertschinger HU, Stranzinger G, Vogeli P: Inheritance of the F4ab, F4ac and F4ad $E$. coli receptors in swine and examination of four candidate genes for F4acR. J Anim Breed Genet 2005, 122(Suppl 1):5-14 
14. Wang $Y$, Ren J, Lan $L$, Yan $X$, Huang $X$, Peng $Q$, Tang H, Zhang B, Ji H, Huang $L$ : Characterization of polymorphisms of transferrin receptor and their association with susceptibility to ETEC F4ab/ac in pigs. J Anim Breed Genet 2007, 124:225-229.

15. Teo M, Tan L, Lim L, Manser E: The tyrosine kinase ACK1 associates with clathrin-coated vesicles through a binding motif shared by arrestin and other adaptors. J Biol Chem 2001, 276:18392-18398.

16. Assoum M, Salih MA, Drouot N, H'Mida-Ben BD, Lagier-Tourenne C, AlDrees A, Elmalik SA, Ahmed TS, Seidahmed MZ, Kabiraj MM, Koenig M: Rundataxin, a novel protein with RUN and diacylglycerol binding domains, is mutant in a new recessive ataxia. Brain 2010, 133:2439-2447.

17. Barrett JC, Fry B, Maller J, Daly MJ: Haploview: analysis and visualization of LD and haplotype maps. Bioinformatics 2005, 21:263-265.

18. Desmet FO, Hamroun D, Lalande M, Collod-Beroud G, Claustres M, Beroud C: Human Splicing Finder: an online bioinformatics tool to predict splicing signals. Nucleic Acids Res 2009, 37:e67.

19. Nygard $A B$, Jorgensen $C B$, Cirera $S$, Fredholm M: Selection of reference genes for gene expression studies in pig tissues using SYBR green qPCR. BMC Mol Biol 2007, 8:67.

20. Vandesompele J, De Preter K, Pattyn F, Poppe B, Van Roy N, De Paepe A, Speleman F: Accurate normalization of real-time quantitative RT-PCR data by geometric averaging of multiple internal control genes. Genome Biol 2002, 3.

21. Zhang B, Ren J, Yan X, Huang X, Ji H, Peng Q, Zhang Z, Huang L: Investigation of the porcine MUC13 gene: isolation, expression, polymorphisms and strong association with susceptibility to enterotoxigenic Escherichia coli F4ab/ac. Anim Genet 2008, 39:258-266.

22. Ji H, Ren J, Yan X, Huang X, Zhang B, Zhang Z, Huang L: The porcine MUC20 gene: molecular characterization and its association with susceptibility to enterotoxigenic Escherichia coli F4ab/ac. Mol Biol Rep 2010, 38:1593-1601.

23. Rasschaert K, Verdonck F, Goddeeris BM, Duchateau L, Cox E: Screening of pigs resistant to $\mathrm{F} 4$ enterotoxigenic Escherichia coli (ETEC) infection. Vet. Microbiol 2007, 123:249-253.

24. Li H, Li Y, Qiu X, Niu X, Liu Y, Zhang Q: Identification and screening of gene(s) related to susceptibility to enterotoxigenic Escherichia coli F4ab/ ac in piglets. AJAS 2008, 21:489-493.

25. Rampoldi $\mathrm{A}$, Jacobsen $\mathrm{MJ}$, Bertschinger $\mathrm{HU}$, Joller $\mathrm{D}$, Burgi $\mathrm{E}$, Vogeli $\mathrm{P}$, Andersson L, Archibald AL, Fredholm M, Jorgensen CB, Neuenschwander S: The receptor locus for Escherichia coli F4ab/F4ac in the pig maps distal to the MUC4-LMLN region. Mamm Genome 2010, 22:122-129.

26. Schroyen M, Stinckens A, Verhelst R, Niewold T, Buys N: Expression of MUC13 and MUC20 in relation to enterotoxigenic E. coli sensitivity in pigs. proceedings of Pig Genome III Conference Hinxton, United Kingdom; 2009.

doi:10.1186/1756-0500-4-225

Cite this article as: Jacobsen et al:: Characterisation of five candidate genes within the ETEC F4ab/ac candidate region in pigs. BMC Research Notes 2011 4:225

\section{Submit your next manuscript to BioMed Central and take full advantage of:}

- Convenient online submission

- Thorough peer review

- No space constraints or color figure charges

- Immediate publication on acceptance

- Inclusion in PubMed, CAS, Scopus and Google Scholar

- Research which is freely available for redistribution 